Ann. Zootech., I969, 18 (I) 4I-54.

\title{
RATIONNEMENT DES VEAUX MÁLES D'ÉLEVAGE AVEC DES QUANTITÉS RÉDUITES DE LAIT DE REMPLACEMENT ET DES QUANTITÉS IMPORTANTES DE LAIT ÉCRÉMÉ FRAIS
}

\author{
(h.-V. BOUCQUE, F.-X. BCYStSE et B.-(i. COT'TYN \\ Station de Recherches de l'Silat pour l'Alimentation du Bétail, \\ Gontrode (Belgique)
}

\section{SOMMAIRE}

Trois groupes de 16 veaux mâles de la race Pie-Ronge de lilandre oricntale ont été éleves suivant différents régimes comprenant des quantités importantes de lait écrémé frais $(564,605$ et 6461$)$ et des quantités réduites de "lait de rcmplacinient " (I9,3, I 4,0 et 8,8 $\mathrm{kg}$ d'aliment d'allaitement) offerts respectivement pendant $8,6 \mathrm{et} 4$ semaines. Outre ces « laits ", les veaux recoivent par animal et par jour, $3 \mathrm{~kg}$ au maximum d'un aliment concentré à faible teneur en matières azotées, et du foin à volonté. Ja faible quantité de "lait de remplacement " consommée par les aninaux des groupes II et III n'a pas défavorablement influencé la croissance (il n'y a pas de différence significative entre les augmentations moyennes de poids durant les i 6 semaines de l'essai). La croissance move'nue pendant les I 2 jours de l'essai est respertivement de 982,908 et $939 \mathrm{~g} / \mathrm{j}$.

Il semble done que de faibles quantités de matières grasses soient sulfisantes pour obtenir une forte augnentation de poids. L“indice de consommation est de $2,4 \mathrm{I}$ et 2,52 unités fourragéres par $\mathrm{kg}$ de gain de poids vif. En tenant compte des prix unitaires actuels des produits alimentaires sur le marché belge, on obtient un coût de l'alimentation d'environ $20 \mathrm{HB}$ par kg de gain. I.es besoins en main-d'ouvre pour la distribution du lait écrémé pendant i 6 semaines influencent cependant défavorablement les frais généraux de ce mode d'élevage.

\section{INTRODUCTION}

Au point de vue anatomique comme physiologique, les veaux nouveau-nés peuvent être considérés comme des monogastriques (WARNIR, FIATT et LoOI.SI, I956) ; il est donc indispensable, durant les premières semaines, de leur offrir des aliments lactés. Parmi les différents produits laitiers pouvant être pris en considération pour l'élevage des veaux, l'éleveur a le choix entre le lait entier, le lait écrémé,

(1) Publication R. V. V. $n^{0}, 46$. 
le babeurre, le lactosérum et le «lait de remplacement ». Dans un grand nombre d'exploitations, on fait appel, après la période du colostrum, à du "lait de remplacement ". D'autres ont recours à l'utilisation de deux produits laitiers, par exemple du lait entier avec du lait écrémé ou du lait de remplacement avec du lait écrémé. Dans une expérience sur 63 veaux femelles de la race Pie-Rouge de Flandre orientale, BuYsSE et MARTIN (I96I) ont obtenu une croissance très satisfaisante, atteignant en moyenne $829,5 \mathrm{~g} / \mathrm{j}$, avec une ration de $\mathrm{I} 651$ de lait entier et de 6401 de lait écrémé frais ; le coît de l'alimentation était cependant relativement élevé $(26,52 \mathrm{FB} / \mathrm{kg})$ aux prix unitaires suivants : 4,75 FB/1 pour le lait entier, I,30 $\mathrm{FB} / 1$ pour le lait écrémé, $5, \$ 0 \mathrm{FB} / \mathrm{kg}$ pour les aliments concentrés et $2 \mathrm{FB} / \mathrm{kg}$ pour le foin. La deuxième combinaison, "lait de remplacement " avec du lait écrémé frais, n'avait jusqu'à présent été expérimentée à la Station que sur un nombre restreint de veaux (Boucque et Buysse, i966).

Le présent article concerne les résultats obtenus avec 48 veaux mâles recevant des quantités de lait écrémé frais importantes en combinaison avec des quantités réduites de "lait de remplacement ". Outre la valorisation de quantités de lait écrémé frais importantes, nous avons étudié l'influence sur la croissance, la quantité d'aliments consommée et le coût de l'alimentation.

\section{MATÉRIEI, ET MÉTHODES}

\section{Animaux}

Iintre septembre 1965 et juin 1966,4 séries de 3 groupes de 4 veaux mâles (race Pie-Rouge de Flandre orientale) ont été achetées à l'âge de 8 à i 2 jours. La période expérimentale s'étend sur I6 semaines. Tous les veaux sont en stabulation libre dans des boxes à paille; 3 séries disposent de boxes individuels; dans la $4^{\mathrm{e}}$ série il y a 4 veaux par box. Ies veaux sont pesés tous les quatorze jours.

\section{Régimes alimentaires}

En vue de la valorisation de quantités importantes de lait écrémé frais, celui-ci a été offert durant toute la période d'élevage de I 6 semaines. Le "lait de remplacement" est donné durant des périodes de 8,6 ou 4 semaines. Les quantités journalières de "lait de remplacement " et de lait écrémé sont combinées de telle sorte que la quantité totale de produits laitiers, exprimée en litres, soit la même pour les trois groupes (tabl. I).

Dans le tableau 2, les trois régimes sont donnés en détail. Les produits laitiers sont distribués deux fois par jour, régulièrement à la même heure. Pour préparer le "lait de remplacement " on emploie de l'eau chaude, de sorte qu'après y avoir ajouté du lait écrémé frais, la température du mélange s'élève encore à $35^{\circ} \mathrm{C}$. La concentration du " lait de remplacement » est portée graduellement de 70 à $125 \mathrm{~g}$ par litre d'eau $(70 \mathrm{~g}+930 \mathrm{~g}$ d'eau etc. $)$.

$A$ partir de la $2^{\mathbf{e}}$ semaine d'expérience, les veaux disposent de foin et d'eau à volonté; un aliment concentré à faible teneur en matières azotées est distribué avec un maximum de $3 \mathrm{~kg}$ par animal et par jour. Au cours de la première semaine expérimentale tous les veaux reçoivent per os $500000 \mathrm{UI}$ de vitamine $A$ et 250000 UI de vitamine $D_{3}$; à cette occasion ils sont vaccinés avec un vaccin polyvalent (Institut sérothérapique de Gembloux, Belgique).

\section{Composition des aliments}

Le tableau 3 donne la composition chimique des aliments. Les veaux des trois groupes recevant du lait écrémé au cours de toute l'expérience, on a fabriqué un aliment concentré à faible teneur en matières azotées, ne contenant pas de protéines d'origine animale. L'aliment concentré est composé des matières premières suivantes (p. 100) : 40 d'orge concassée, 20 de farine de mais, 12 d'avoine concassée, ro de farine de lin, ro de tourteau de soja (extraction : $44 \mathrm{p}$. тoo), 5 de sucre dénaturé, $\mathrm{I}, 5$ de phosphates alimentaires, 0,5 de craie, 0,5 de chlorure de sodium, des oligoéléments ( $5 \circ \mathrm{g}$ de 
TABLEAU I

\section{Rationnement}

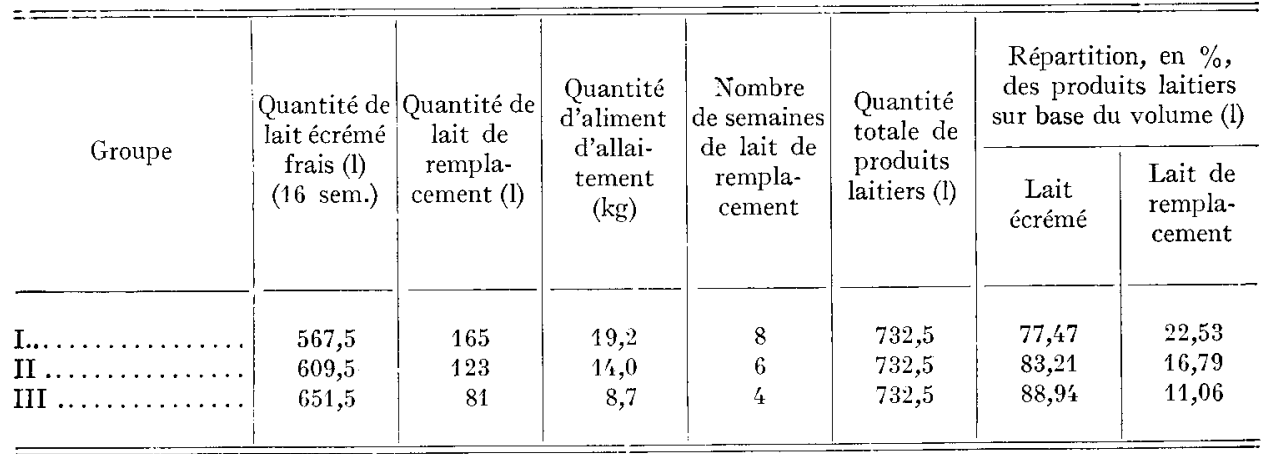

TABLEAU 2

Modalités de distribution du lait de remplacement et du lait écrémé frais (2 repas par jour)

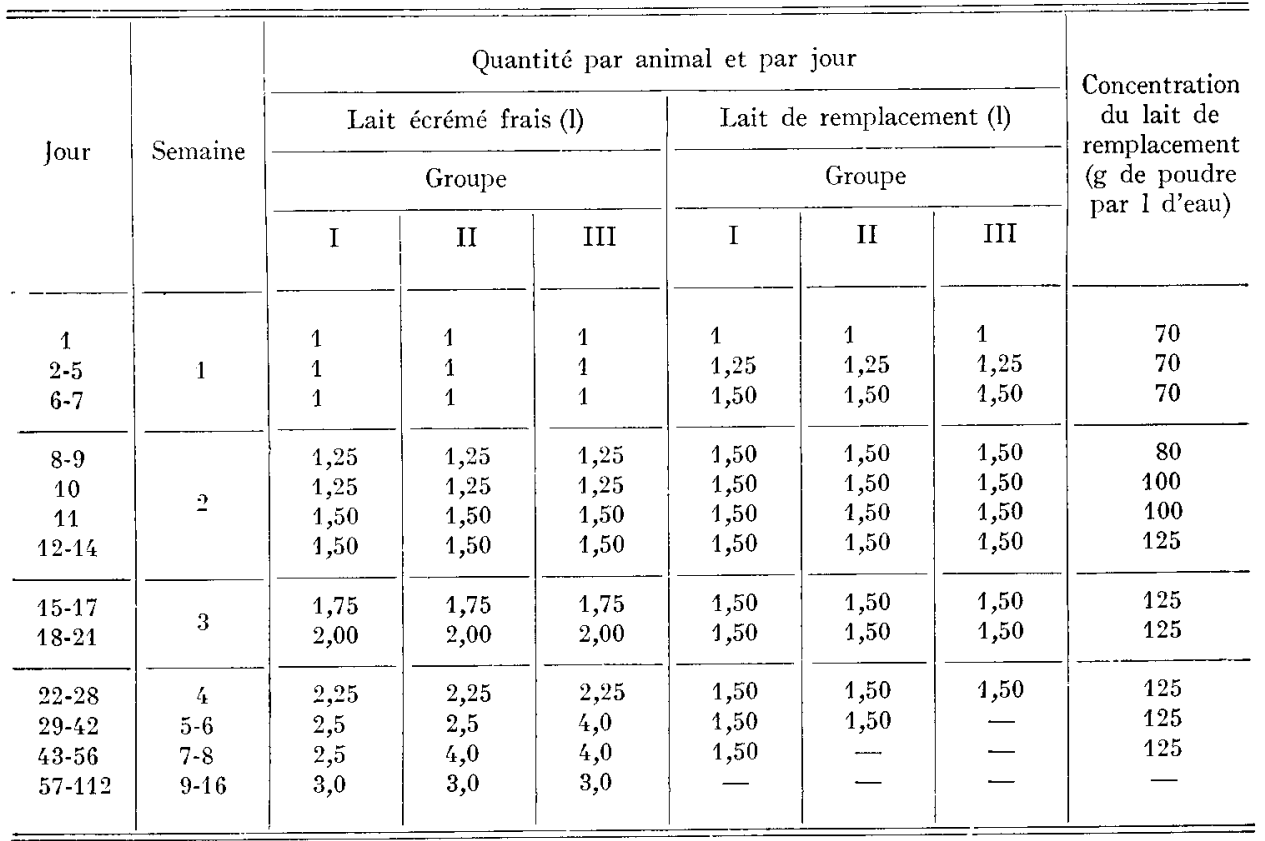


$\mathrm{FeSO}_{4},{ }_{7} \mathrm{H}_{2} \mathrm{O} ; 30 \mathrm{~g}$ de $\left(\mathrm{CuSO}_{4},{ }_{5} \mathrm{H}_{2} \mathrm{O} ; 50 \mathrm{~g}\right.$ de $\mathrm{MnSO}_{4}, \mathrm{II}_{2} \mathrm{O}$; Io g de $\mathrm{CoSO}_{4}, 7 \mathrm{H}_{2} \mathrm{O}$; $220 \mathrm{~g}$ de $7 n \mathrm{SO}_{4}$, ${ }_{7} \mathrm{H}_{2} \mathrm{O}$ et $5 \mathrm{~g}$ de $\mathrm{KI}$ par tonne d'aliment concentré) et des vitamines (IO 000 UI de vit. $\lambda$ et 3400 UI de vit. $\mathrm{D}_{3}$ par $\mathrm{kg}$ d'aliment (oncentré).

Le lait écrémé frais est livré tous les jours par une laiterie. L'aliment d'allaitement est un aliment industriel et le foin est livré par le domaine expérimental.

\section{TABIEAU 3}

Composition chimique des aliments

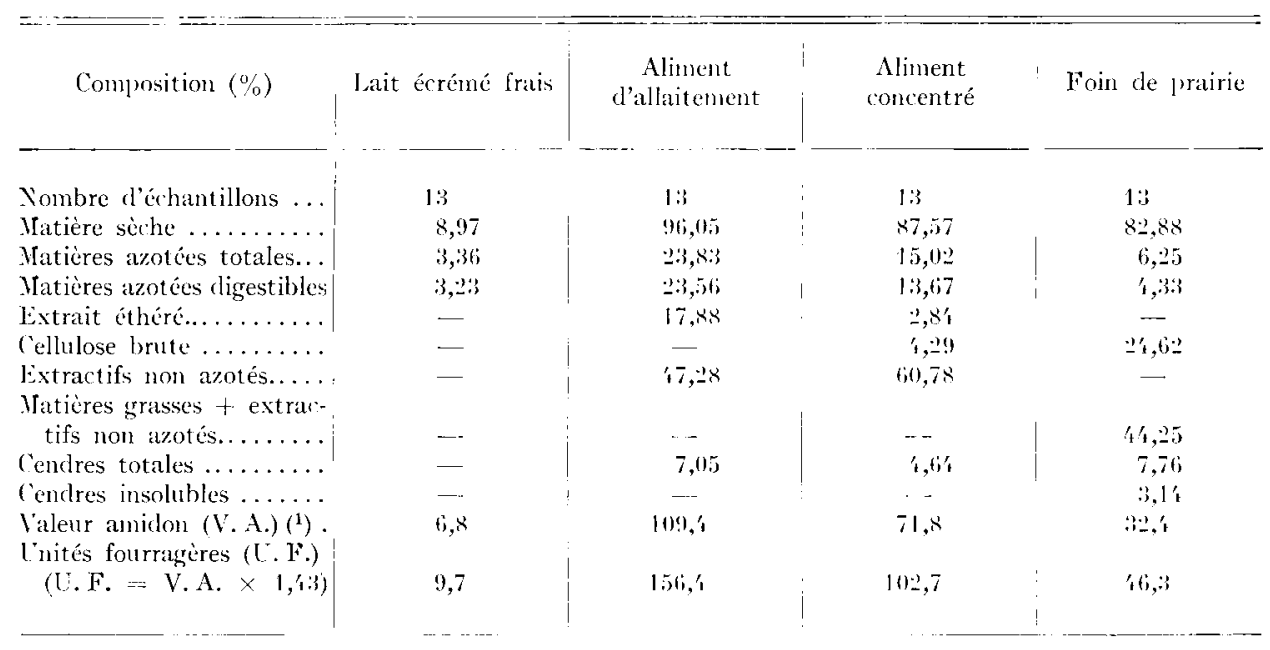

(1) Calculée d'aprés KeLL.Nik (I96ii). Coefficients de digestibilité utilisés :

- ponr les matières azotées : test de laboratoire pejsine - HCl.

- pour les matiòres grasses : dans le lait de remplatement :95 dans les aliments concentrís : 90

- pour la cellulose brute: dans les aliments concentrés : i3 dans le foin de prairie : til

- pour les extractifs non azotés : dans le lait de remulacement : 98 dans laliment concentré : :90 dams le foin de prairie : 6 i:

\section{RÉSULTATS ET DISCUSSION}

\section{Croissance}

Le tableau 4 et la figure I représentent l'évolution du poids au cours des I6 semaines d'expériences. Au tableau 5 figure l'augmentation en poids par période de 2 ou de 4 semaines. L'augmentation moyenne de poids chez les veaux du groupe I dépasse de $8,2 \mathrm{p}$. Ioo celle du groupe II et de $4,6 \mathrm{p}$. Ioo celle du groupe III (I Io,o kg pour le groupe I, contre IOI, $7 \mathrm{~kg}$ pour le groupe II et $105,2 \mathrm{~kg}$ pour le groupe III). De l'analyse statistique des résultats (analyse de variance), il ressort cependant que 
TABIEAU 4

Poids des animaux pendant la période d'essai (48 veaux mâles)

\begin{tabular}{|c|c|c|c|}
\hline \multirow[b]{2}{*}{$\begin{array}{l}\text { Durée de l'essai } \\
\text { (semaines) }\end{array}$} & \multicolumn{3}{|c|}{ Poids vif $(\mathrm{kg})$, moyenne et écart-type } \\
\hline & $\begin{array}{l}\text { Groupe } 1 \\
\text { (8 semaines de lait } \\
\text { de remplacement) }\end{array}$ & $\begin{array}{c}\text { Groupe } 2 \\
\text { (6 semaines de lait } \\
\text { de remplacoment) }\end{array}$ & $\begin{array}{c}\text { Groupe } 3 \\
\text { (4 semaines de lait } \\
\text { de remplacement) }\end{array}$ \\
\hline $0(\mathbf{1})$ & $49,6 \pm 1,9$ & $50,1 \neq 5,3$ & $48,9 \pm 6,6$ \\
\hline 2 & $54,4 \pm 5,5$ & $53,5 \pm 5,8$ & $53,2 \pm 7,0$ \\
\hline 4 & $66,2 \pm 6,7$ & $65.1 \pm 7,3$ & $6^{\prime}, 9 \pm 9,1$ \\
\hline 6 & $80,6 \pm 7,3$ & $78.0 \pm 8,7$ & $76,8 \pm 12.3$ \\
\hline 8 & $96,3 \pm 9,7$ & $91,8 \pm 10,1$ & $90,1 \pm 14,2$ \\
\hline 10 & $112,1 \pm 11,1$ & $105,4 \pm 12, k$ & $106,1 \pm 18,1$ \\
\hline 12 & $198,2 \pm 13,9$ & $121,0 \pm 15,5$ & $121,9 \pm 21,4$ \\
\hline $1 / 4$ & $142,9 \pm 16,7$ & $136,1 \pm 16,1$ & $138,3 \pm 23,1$ \\
\hline 16 & $159,6 \pm 19,3$ & $151,8 \pm 17,7$ & $154,1 \pm 24,5$ \\
\hline Nombre de veaux. & 16 & 16 & 16 \\
\hline
\end{tabular}

(1) Au debut de l'essai les veaux avaient déjà 8 à 12 jours.

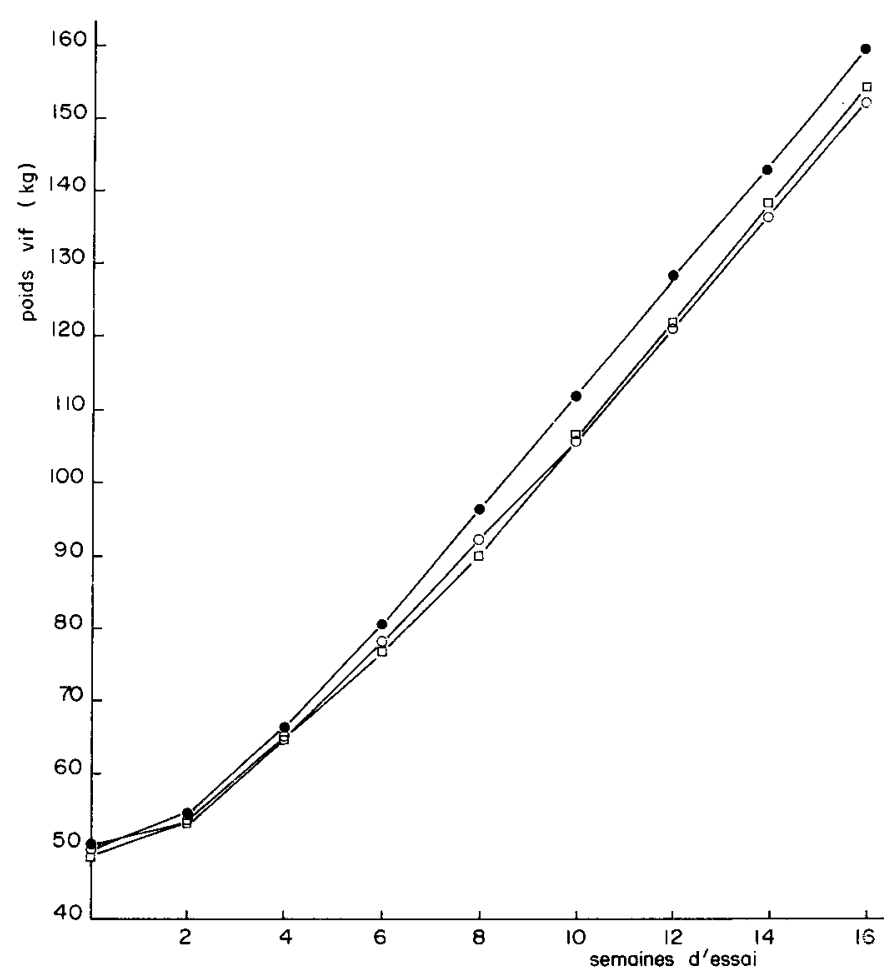

FIG. I. - Courbe de croissance des veaux mâles

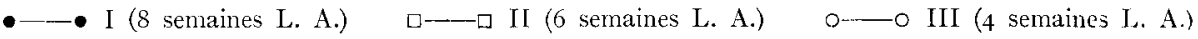


cette différence n'est pas significative $(F$ calculé $=\mathrm{I}, 57$ et $\mathrm{F}$ théorique $: 2 \cdot 30=3,32$ pour $\mathrm{P}=0,05)$ (SNEDECOR I956).

Dans ces régimes comprenant durant I6 semaines du lait écrémé frais, la limitation de la quantité d'aliment d'allaitement (8,8 $\mathrm{kg}$ par animal, pour 4 semaines), n'a pas exercé d'influence significative sur la croissance. L'écart au sein même des groupes expérimentaux est toutefois important. Le coefficient de variation, calculé sur l'augmentation en poids pendant les 16 semaines d'expérience, s'élève pour les trois groupes à $14,5,14,7$ et I9,3 p. Ioo respectivement.

\section{TABI,EAU 5}

Augmentation moyenne de poids et croissance journalière par période $\mathrm{A}=$ augmentation de poids ( $\mathrm{kg} /$ période) (moyenne et écart-type) $\mathrm{B}=$ gain de poids $(\mathrm{g} / \mathrm{j})$ (moyenne et écart-type)

\begin{tabular}{|c|c|c|c|c|}
\hline $\begin{array}{c}\text { Périodes } \\
\text { expérimentales } \\
\text { (en semaines) }\end{array}$ & & $\begin{array}{c}\text { Groupe I } \\
\text { (8 semaines de lait } \\
\text { de remplacement) }\end{array}$ & $\begin{array}{l}\text { Groupe II } \\
\text { (6 semaines de lait } \\
\text { de remplacement) }\end{array}$ & $\begin{array}{c}\text { Groupe III } \\
\text { ('t semaines de lait } \\
\text { de remplacement) }\end{array}$ \\
\hline $0-4$ & $\begin{array}{l}\mathrm{A} \\
\mathrm{B}\end{array}$ & 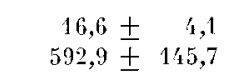 & $\begin{array}{r}15,0 \pm \quad 4,1 \\
535,7 \pm \quad 144,6\end{array}$ & $\begin{array}{r}16,0 \pm \quad 4,3 \\
571,4 \pm 152,9\end{array}$ \\
\hline $5-6$ & $\begin{array}{l}\mathrm{A} \\
\mathrm{B}\end{array}$ & $\begin{array}{r}14,4 \pm \quad 1,5 \\
1028,6 \pm 107,2\end{array}$ & $\begin{array}{r}12,9 \pm \quad 3,0 \\
921,4 \pm \quad 212,6\end{array}$ & $\begin{array}{r}11,9 \pm \quad 4,3 \\
850,0 \pm 308,5\end{array}$ \\
\hline $7-8$ & $\begin{array}{l}\mathrm{A} \\
\mathrm{B}\end{array}$ & $\begin{array}{r}15,7 \pm \quad 4,7 \\
1121,4 \pm 336,8\end{array}$ & $\begin{array}{r}13,8 \pm \quad 3,5 \\
985,7 \pm 246,4\end{array}$ & $\begin{array}{r}13,3 \pm \quad 5,0 \\
950,0 \pm 355,2\end{array}$ \\
\hline $9-12$ & $\begin{array}{l}\text { A } \\
\text { B }\end{array}$ & $\begin{array}{r}31,9 \pm \quad 5,2 \\
1139,3 \pm 186,5\end{array}$ & $\begin{array}{r}29,2 \pm \quad 6,5 \\
1042,9 \pm 230,4\end{array}$ & $\begin{array}{r}31,8 \pm \quad 8,8 \\
1135,7 \pm 315,1\end{array}$ \\
\hline $13-16$ & $\begin{array}{l}\mathrm{A} \\
\mathrm{B}\end{array}$ & $\begin{array}{r}31,4 \pm \quad 7,9 \\
1121,4 \pm 281,8\end{array}$ & $\begin{array}{r}30,8 \pm \quad 5,1 \\
1100,0 \pm 194,1\end{array}$ & $\begin{array}{r}32,2 \pm \quad 5,9 \\
1150,0 \pm 210,3\end{array}$ \\
\hline $0-6$ & $\begin{array}{l}\mathrm{A} \\
\mathrm{B}\end{array}$ & $\begin{array}{r}31,0 \pm \quad 4,5 \\
738,1 \pm 107,2\end{array}$ & $\begin{array}{r}27,9 \pm \begin{array}{r}5,6 \\
66\end{array}, 3 \pm 132,6\end{array}$ & $\begin{array}{r}27,9 \pm 7,1 \\
664,3 \pm 170,0\end{array}$ \\
\hline 0.8 & $\begin{array}{l}\mathrm{A} \\
\mathrm{B}\end{array}$ & $\begin{array}{r}46,7 \pm \quad 6,7 \\
833,9 \pm 119,8\end{array}$ & $\begin{array}{r}41,7 \pm \quad 7,4 \\
744,6 \pm 131,6\end{array}$ & $\begin{array}{r}41,2 \pm \quad 9,9 \\
735,7 \pm 176,0\end{array}$ \\
\hline $0-12$ & $\begin{array}{l}\text { A } \\
\text { B }\end{array}$ & $\begin{array}{r}78,6 \pm 11,2 \\
935,7 \pm 133,8\end{array}$ & $\begin{array}{r}70,9 \pm 12,5 \\
84,0 \pm 148,3\end{array}$ & $\begin{array}{r}73,0 \pm \quad 17,3 \\
869,0 \pm 205,3\end{array}$ \\
\hline $0-16$ & $\begin{array}{l}\mathrm{A} \\
\mathrm{B}\end{array}$ & $\begin{array}{l}110,0 \pm 15,9 \\
982,1 \pm 141,8\end{array}$ & $\begin{array}{l}101,7 \pm \quad 15,0 \\
908,0 \pm 133,9\end{array}$ & $\begin{array}{r}105,2 \pm \quad 20,3 \\
939,3 \pm 181,4\end{array}$ \\
\hline
\end{tabular}

Le gain de poids, qui est en moyenne de $982, \mathrm{I}, 908,0$ et $939,3 \mathrm{~g} / \mathrm{j}$ peut être considéré comme très élevé. D'après KRUGER (r957 b) et MATHIEU et WEGAT-LITRE (I96r), il faut que la croissance au cours de la période d'élevage soit au minimum de $700 \mathrm{~g} / \mathrm{j}$ si 1'on veut avoir la garantie que le développement ultérieur sera suffisant. Différents chercheurs obtiennent de telles croissances avec des régimes alimentaires dans lesquels des quantités de lait écrémé frais importantes sont distribuées avec des quan- 
TABLEAU 6

Quantité d'aliments consommée ( $\mathrm{kg}$ par période et par veau)

\begin{tabular}{|c|c|c|c|c|}
\hline $\begin{array}{l}\text { Période expérimentale } \\
\text { (semaines) }\end{array}$ & Aliments $\left({ }^{1}\right)$ & Groupe I & Groupe II & Groupe III \\
\hline $0-1$ & $\begin{array}{ll}\text { L. E. F. }(\mathrm{l}) \\
\text { A. A. } & (\mathrm{kg}) \\
\text { Conc. } & (\mathrm{kg}) \\
\text { Foin } & (\mathrm{kg})\end{array}$ & $\begin{array}{r}89,02 \\
8,79 \\
6,68 \\
0,75\end{array}$ & $\begin{array}{r}88,88 \\
8,79 \\
6,67 \\
0,52\end{array}$ & $\begin{array}{r}89,06 \\
8,81 \\
6,73 \\
0,68\end{array}$ \\
\hline $5-6$ & $\begin{array}{l}\text { L. L. F. (l) } \\
\text { A. A. }(\mathrm{kg}) \\
\text { Conc. }(\mathrm{kg}) \\
\text { Foin }(\mathrm{kg})\end{array}$ & $\begin{array}{r}69,88 \\
5,24 \\
11,71 \\
1,51\end{array}$ & $\begin{array}{r}69,89 \\
5,24 \\
9,73 \\
1,07\end{array}$ & $\begin{array}{c}110, t^{\prime} \\
\frac{-}{10,14} \\
1,6 t\end{array}$ \\
\hline $7-8$ & $\begin{array}{lr}\text { L. E. F. } & (\mathrm{l}) \\
\text { A. A. } & (\mathrm{kg}) \\
\text { Conc. } & (\mathrm{kg}) \\
\text { Foin } & (\mathrm{kg})\end{array}$ & $\begin{array}{r}70,00 \\
5,25 \\
17,84 \\
1,90\end{array}$ & $\begin{array}{c}112,00 \\
- \\
17,26 \\
2,17\end{array}$ & $\begin{array}{c}112,00 \\
- \\
16,02 \\
2,22\end{array}$ \\
\hline $9-12$ & $\begin{array}{l}\text { L. E. F. (1) } \\
\text { Conc. }(\mathrm{kg}) \\
\text { Foin }(\mathrm{kg})\end{array}$ & $\begin{array}{r}167,13 \\
61,26 \\
6,12\end{array}$ & $\begin{array}{r}166,81 \\
58,73 \\
5,81\end{array}$ & $\begin{array}{r}166,97 \\
60,87 \\
6,51\end{array}$ \\
\hline $13-16$ & $\begin{array}{l}\text { L. E. F. (l) } \\
\text { Conc. }(\mathrm{kg}) \\
\text { Foin }(\mathrm{kg})\end{array}$ & $\begin{array}{r}167,63 \\
74,37 \\
10,36\end{array}$ & $\begin{array}{r}167,56 \\
74,27 \\
11,58\end{array}$ & $\begin{array}{r}167,75 \\
73,10 \\
13,81\end{array}$ \\
\hline $0-6$ & $\begin{array}{l}\text { L. E. F. }(\mathrm{l}) \\
\text { A. A. }(\mathrm{kg}) \\
\text { Conc. }(\mathrm{kg}) \\
\text { Foin } \quad(\mathrm{kg})\end{array}$ & $\begin{array}{r}158,89 \\
14,03 \\
18,39 \\
2,25\end{array}$ & $\begin{array}{r}158,76 \\
14,03 \\
16,110 \\
1,59\end{array}$ & $\begin{array}{r}199,47 \\
8,81 \\
16,87 \\
2,33\end{array}$ \\
\hline $0-8$ & $\begin{array}{lr}\text { L. E. F. }(\mathrm{l}) \\
\text { A. A. }(\mathrm{kg}) \\
\text { Conc. } & (\mathrm{kg}) \\
\text { Foin } \quad(\mathrm{kg})\end{array}$ & $\begin{array}{r}228,89 \\
19,28 \\
36,24 \\
4,15\end{array}$ & $\begin{array}{r}270,76 \\
14,03 \\
33,66 \\
3,76\end{array}$ & $\begin{array}{r}311,47 \\
8,81 \\
32,89 \\
4,55\end{array}$ \\
\hline $0-12$ & $\begin{array}{l}\text { L. I. F. }(1) \\
\text { A. A. }(\mathrm{kg}) \\
\text { Conc. }(\mathrm{kg}) \\
\text { Foin }(\mathrm{kg})\end{array}$ & $\begin{array}{r}396,02 \\
19,28 \\
97,49 \\
10,27\end{array}$ & $\begin{array}{r}437,58 \\
14,03 \\
92,39 \\
9,57\end{array}$ & $\begin{array}{r}478,1^{\prime} \\
8,81 \\
93,76 \\
11,06\end{array}$ \\
\hline $0-16$ & $\begin{array}{lr}\text { L. E. F. (l) } \\
\text { A. A. } & (\mathrm{kg}) \\
\text { Conc. } & (\mathrm{kg}) \\
\text { Foin } & (\mathrm{kg})\end{array}$ & $\begin{array}{r}563,6^{\prime} \\
19,28 \\
171,86 \\
20,63\end{array}$ & $\begin{array}{r}605,14 \\
14,03 \\
166,66 \\
21,15\end{array}$ & $\begin{array}{r}646,19 \\
8,81 \\
166,86 \\
24,87\end{array}$ \\
\hline
\end{tabular}

(l) L. E. F. = Lait écrémé frais ; A. A. = Aliment d'allaitement ; Conc. = Aliment concentré. 
tités de lait entier limitées. Pour une période d'élevage de I5 semaines, Richter et al. (I957) ont obtenu une croissance de $790 \mathrm{~g} / \mathrm{j}$ avec 2091 de lait entier et 613 1 de lait écrémé. Hvidstén et Hor.E (I962) ont obtenu une croissance de $879 \mathrm{~g} / \mathrm{j}$ avec 1301 de lait entier et 7951 de lait écrémé au cours d'une période de 18 semaines. Avec 9I 1 de lait entier et 7671 de lait écrémé acidifié, les mêmes auteurs (I965) ont obtenu un gain de poids de $769 \mathrm{~g} / \mathrm{j}$. Opstvis'r ( 1964 ) a obtenu une croissance moyenne très élevée de I oog $g$ avec seulement 431 de lait entier et 6771 de lait écrémé. L'expérience avait duré $\mathrm{I} 6$ semaines.

I)ans toutes ces expériences, les veaux disposaient également de foin et d'aliments concentrés à volonté.

Iin ce qui concerne l'augmentation de poids par période, l'on peut observer que durant les deux premières semaines faisant suite à la suppression du lait de remplacement, c'est-à-dire, après 4 semaines dans le groupe III et après 6 semaines dans le groupe II, il se produit un ralentissement temporaire de la croissance par rapport at groupe I. A partir de la $g^{\mathrm{e}}$ semaine, le rythme de la croissance est pratiquement le même dans les trois groupes.

\section{Quantité d'aliments consommée}

Le tableau 6 donne, par période et pour toute la durée de l'expérience, la quantité des divers aliments consommée. On peut constater que la suppression du lait de remplacement, après 4 semaines dans le groupe III ou après 6 semaines dans le groupe II, n'a pas influencé sensiblement la quantité d'aliments concentrés et de foin consommée au cours des semaines suivantes. Ceci s'explique car, chaque fois que 1a quantité de lait de remplacement est diminuée, la quantité de lait écrémé frais est augmentée, de sorte que chaque groupe reçoit un même volume de produits laitiers (tabl. 2).

La substitution du " lait de remplacement " par du lait écrémé frais donne lieu à une plus faible quantité d'énergie consommée, ce qui ressort des gains de poids, qui, dans les groupes II et III, sont quelque peu inférieurs à ceux enregistrés dans le groupe I. La quantité d'aliment concentré et cle foin consommée pour l'ensemble de la périocle expérimentale est pratiquement la même clans les trois groupes.

La (quantité d'aliment concentré et de foin consommée (tabl. 7), se situe, dans les trois régimes comprenant du lait écrémé en abondance, à un niveau beaucoup plus bas que dans des régimes renfermant des quantités restreintes de produits laitiers. MaTHIit et WrGaT-LiTris (Ig6r et ig62) constatent que la quantité d'aliment concentré consommée varie en raison inverse de la quantité de lait offerte ; la quantité de foin consommée est également moindre chez les veaux sevrés à un âge plus précoce; cette quantité augmente toutefois lorsque l'aliment concentré est limité. Chez I6 veaux mâles sevrés après 5 semaines, Boucoul et Buyssi ( 1967 ) trouvent, au cours de la $6^{\mathrm{e}}$ semaine, une quantité d'aliment concentré de $1,26 \mathrm{~kg} / \mathrm{j}$, alors que les veaux qui reçoivent encore du lait écrémé ne consomment en moyenne quie $0, \$+\mathrm{kg} / \mathrm{j}$. Avec un régime d'élevage combiné, basé sur I50 1 de lait entier et 5501 de lait écrémé, KRUGFr ( 1957 a) note une consommation moyenne de foin de $73 \mathrm{~kg}$ au cours d'une expérience de 15 semaines ; la quantité d'aliment concentré avait toute- 
fois été réduite à $92 \mathrm{~kg}$. Au cours de la I $^{\mathbf{e}}$ semaine, ESCHENBRENNER (I963) trouve, pour une quantité consommée de $I, 5 \mathrm{~kg} / \mathrm{j}$ d'aliment concentré, une quantité de $I, 5 \mathrm{~kg} / \mathrm{j}$ de foin. Il ressort du tableau 7 que ces rapports sont tout autres lorsque les aliments concentrés sont donnés à volonté. Au cours de la $\mathrm{I}^{\mathrm{e}} \mathrm{semaine,} \mathrm{la} \mathrm{quantité}$ d'aliment concentré consommée est d'environ $2,79 \mathrm{~kg} / \mathrm{j}$, alors que la quantité de foin n'atteint que $0,52 \mathrm{~kg} / \mathrm{j}$.

\section{TABLEAU 7}

Quantité d'aliment concentré et de foin consommée ( $\mathrm{kg}$ par veau et par semaine)

\begin{tabular}{|c|c|c|c|c|c|c|}
\hline \multirow{2}{*}{$\begin{array}{c}\text { Semaine } \\
\text { expérimentale }\end{array}$} & \multicolumn{2}{|c|}{ Groupe I } & \multicolumn{2}{|c|}{ Groupe II } & \multicolumn{2}{|c|}{ Groupe III } \\
\hline & Concentré & Foin & Concentré & Foin & Concentré & Foin \\
\hline 1 & - & 一 & - & - & - & — \\
\hline 2 & 0,19 & 0,01 & 0,18 & 0,01 & 0,19 & 0,02 \\
\hline 3 & 0,31 & 0,03 & 0,32 & 0,01 & 0,31 & 0,03 \\
\hline śt & 0,10 & 0,07 & 0,45 & 0,06 & 0,16 & 0,05 \\
\hline 5 & 0,73 & 0,08 & 0,61 & 0,06 & 0,66 & 0,11 \\
\hline 6 & 0,95 & 0,13 & 0,78 & 0,09 & 0,79 & 0,12 \\
\hline 7 & 1,19 & 0,12 & 1,10 & 0,14 & 1,04 & 0,15 \\
\hline 8 & 1,36 & 0,15 & 1,36 & 0,17 & 1,25 & 0,17 \\
\hline 9 & 1,86 & 0,21 & 1,76 & 0,20 & 1,80 & 0,20 \\
\hline 10 & 2,12 & 0,20 & 2,02 & 0,17 & 2,08 & 0,24 \\
\hline 11 & 2,28 & 0,23 & 2,16 & 0,22 & 2,31 & 0,23 \\
\hline 12 & 2,49 & $0,2 z_{k}$ & 2,45 & $0,2 ?_{\mathrm{k}}$ & 2,51 & 0,27 \\
\hline 13 & 2,53 & 0,28 & 2,55 & 0,30 & 2,51 & $0, ; 1$ \\
\hline $1 / t$ & 2,60 & 0,31 & 2,61 & 0,40 & 2,68 & 0,42 \\
\hline 15 & 2,63 & 0,38 & 2,63 & 0,46 & 2,58 & 0,58 \\
\hline 1.6 & 2,87 & 0,51 & 2,83 & 0,49 & 2,67 & 0,57 \\
\hline
\end{tabular}

\section{Indice de consommation et quantité d'éléments nutritifs consommée.}

En se basant sur les données des tableat1x 5 et 6 on peut, pour les différentes périodes expérimentales, calculer la quantité d'aliment consommée par $\mathrm{kg}$ de gain de poids. Dans le tableau 8 ne figure toutefois que l'indice de consommation moyen pour la durée totale de l'expérience. I1 ressort nettement de ce tableau que, dans des

TABIEAU 8

Indice de consommation (I I 2 jours d'essai)

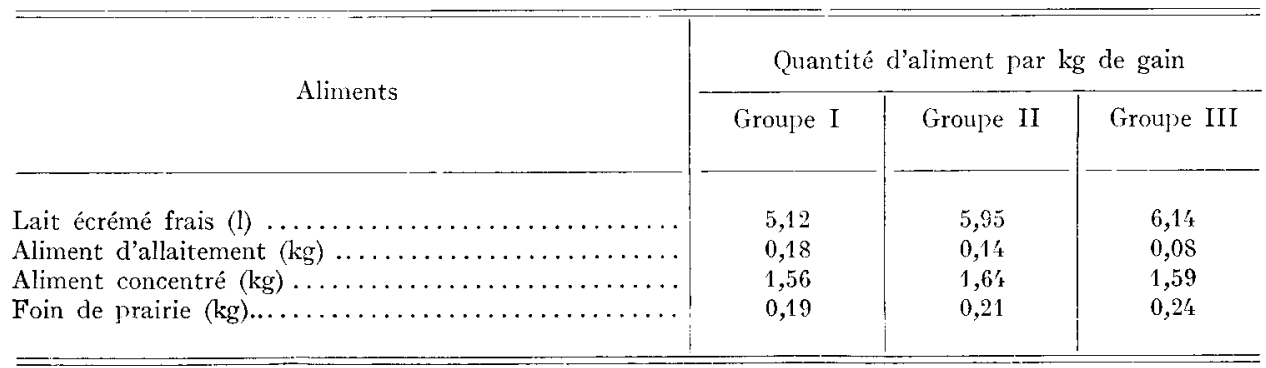


régimes d'élevage, faisant appel à des quantités de produits laitiers importantes, la quantité d'aliments concentrés est faible; elle est moindre que dans des régimes comportant un sevrage précoce. Dans une expérience de sevrage à 1'âge de 5 semaines, BouCQUE et BUYSSE (I967) trouvent, pour une période expérimentale de I6 semaines, une quantité d'aliment concentré de 2,3I kg par $\mathrm{kg}$ de gain ; EECKHOUT et BuYssE (I967) trouvent en moyenne 2 , I I $\mathrm{kg}$ lorsque les veaux sont sevrés à l'âge de 8 semaines, alors que pour notre expérience, dans laquelle les veaux sont sevrés à l'âge de I6 semaines, la quantité d'aliment concentré par $\mathrm{kg}$ de gain a été ramenée à $\mathrm{I}, 60 \mathrm{~kg}$. La composition chimique des aliments (tabl. 3) nous permet de calculer la quantité d'éléments nutritifs ingérés. Le remplacement partiel, dans les groupes II et III, du "lait de remplacement " par un volume égal de lait écrémé frais a entraîné une diminution de matière sèche et d'énergie consommées.

Cette quantité moindre d'énergie, qui est due au fait que les deux groupes reçoivent moins de matières grasses, n'a pas été compensée par une consommation accrue d'aliments secs (aliment concentré et foin), celle-ci étant à peu près la même dans les trois groupes. Il en résulte finalement une augmentation en poids légèrement inférieure dans les groupes II et III que dans le groupe I, bien que cette différence ne soit pas significative. Le gain de poids élevé, $939 \mathrm{~g} / \mathrm{j}$, obtenu avec un régime n'apportant des quantités importantes de matières grasses qu'au cours des quatre premières semaines, vient confirmer la conclusion de Czako (I964) : les besoins en matières grasses des veaux, aussi bien durant la période du régime lacté que durant celle où le régime est composé d'autres aliments, sont très inférieures aux quantités admises habituellement.

TABLEEAU 9

Quantité d'éléments nutritifs consommée

\begin{tabular}{|c|c|c|c|}
\hline \multirow{2}{*}{ Éléments nutritifs } & Groupe I & Groupe II & Groupe III \\
\hline & \multicolumn{3}{|c|}{ Pendant 112 jours d'essai } \\
\hline 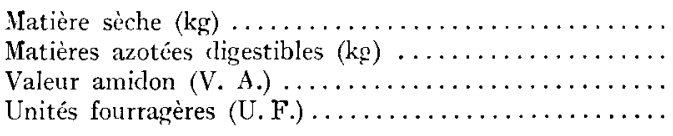 & $\begin{array}{r}236,68 \\
47,13 \\
189,50 \\
270,87\end{array}$ & $\begin{array}{r}231, \geq 3 \\
46,56 \\
183,01 \\
261,59\end{array}$ & $\begin{array}{r}233,15 \\
46, \times 1 \\
181,45 \\
259,31\end{array}$ \\
\hline & \multicolumn{3}{|c|}{ Par $k_{r r}$ de gain de poids } \\
\hline Natière sèche $(\mathrm{kr})$ & 2.16 & 2,27 & $2,2=2$ \\
\hline Matières azotées digestibles $(\mathrm{k}(\mathrm{r})$.. & 0,43 & 0,45 & 0,45 \\
\hline 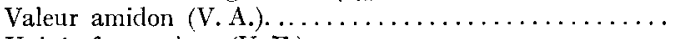 & 1,72 & 1,80 & 1,72 \\
\hline 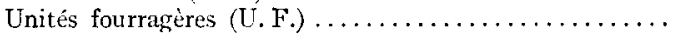 & 2,46 & 2,57 & 2,17 \\
\hline
\end{tabular}

L'on peut déduire du tableau 9 qu'avec 2,46 à 2,57 unités fourragères par $\mathrm{kg}$ de gain, notre expérience a donné un indice de consommation satisfaisant. Cette bonne utilisation de l'énergie est due à la forte croissance journalière. L,es coefficients de 
digestibilité employés (tabl. 3) correspondent très bien à ceux trouvés expérimentalement avec des veaux à l'engrais par MATHIEU et BARRÉ (I964). D'autres chercheurs ont obtenu de bons indices de consommation avec des régimes comprenant des quantités de lait écrémé importantes. Les expériences de HVidsTEN et Holis (I962, I965) et OPSTVEDT (I964) ont donné des indices de consommation de 2,44, 2,55 et 2,67 unités fourragères par $\mathrm{kg}$ de gain.

La quantité d énergie consommée, exprimée en valeur amidon, s'élève pour les trois groupes expérimentaux, à I $692 \mathrm{~g} / \mathrm{j}$, I $634 \mathrm{~g} / \mathrm{j}$ et I $620 \mathrm{~g} / \mathrm{j}$. D'après la norme de KRUGER (I957 b), les besoins en valeur amidon pour l'intervalle de poids obtenu, sont respectivement de I $546 \mathrm{~g}$, I $509 \mathrm{~g}$ et I 5 I $5 \mathrm{~g}$, de sorte que les besoins des trois groupes ont été amplement couverts. D'après KRUGER (I957 b), le rapport optimal entre les matières azotées et la valeur amidon se situe à $I: 4$ pendant les premières semaines, et après $I: 5$ même à $I: 6$. En se basant sur le rapport $I: 4$, les besoins en matières azotées sont de $387 \mathrm{~g} / \mathrm{j}, 377 \mathrm{~g} / \mathrm{j}$ et $378 \mathrm{~g} / \mathrm{j}$. La quantité de matières azotées s'élève pour les trois groupes à $42 \mathrm{I} \mathrm{g} / \mathrm{j}, 4 \mathrm{I} 6 \mathrm{~g} / \mathrm{j}$ et $4 \mathrm{I} 8 \mathrm{~g} / \mathrm{j}$, de sorte que les besoins journaliers en protéines sont eux aussi amplement couverts.

\section{TABIEAU IO}

Performances des veaux (valeurs moyennes et erreur standard) et nourriture ingérée pendant 16 semaines d'élevage au lait écrémé frais, lait de remplacement et concentré

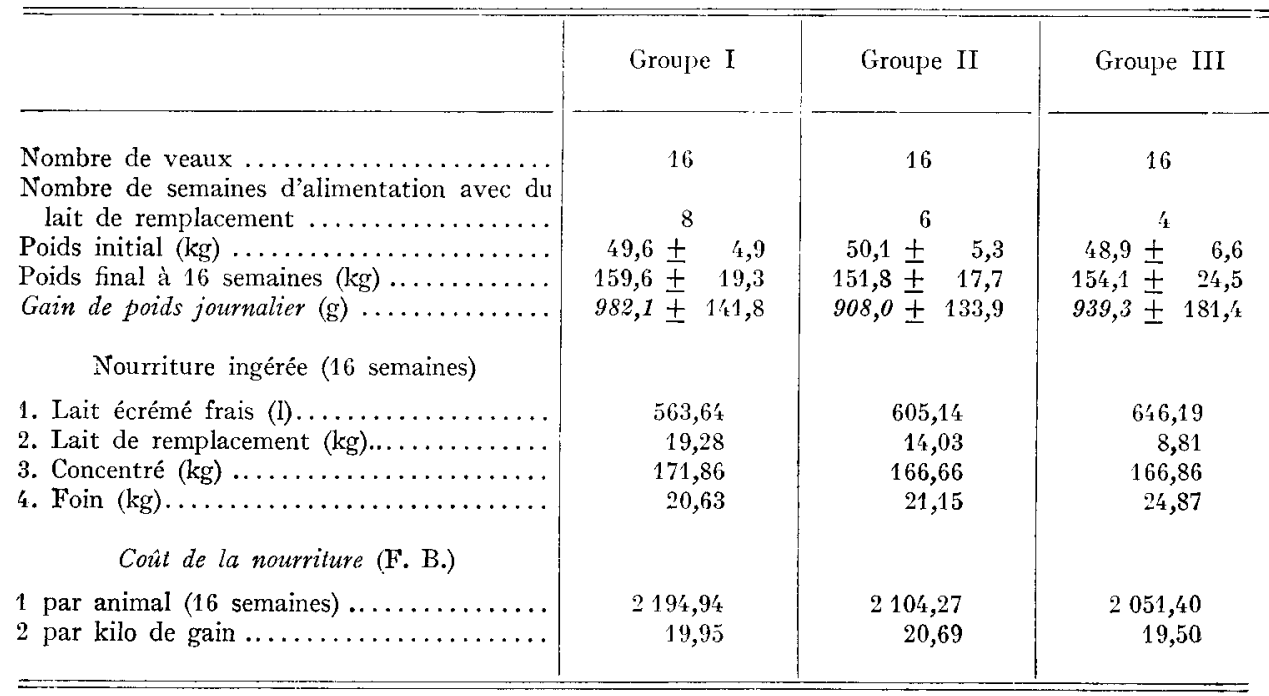

\section{Aspect économique}

Afin d'étudier si des régimes comportant des quantités de lait écrémé frais importantes, sont économiquement justifiés, on peut calculer le prix de revient de l'alimentation. En se basant sur les prix unitaires actuellement en cours en Belgique : $22 \mathrm{FB} / \mathrm{kg}$ pour l'aliment d'allaitement, I,30 FB/1 pour le lait écrémé frais $5,80 \mathrm{FB} / \mathrm{kg}$ pour les concentrés à $I 3,7 \mathrm{p}$. Ioo de p. b. d. (dans la formule il n'y a pas de 
protéines animales) et $2 \mathrm{FB} / \mathrm{kg}$ pour le foin, le prix de revient de l'alimentation par $\mathrm{kg}$ de gain se situe à I9,95 FB, 20,69 FB et I9,50 FB. Il se situe au même niveau que celui qui fut trouvé par Boucoue et Buysse ( 1967 ) avec des veaux sevrés à un âge précoce. Cependant, lorsqu'on considère l'aspect main-d'œuvre, une méthode d'élevage prévoyant l'administration de produits laitiers pendant I 6 semaines devient moins intéressante. Un autre facteur qu'il convient de ne pas perdre de vue est la qualité instable du lait écrémé frais en période estivale.

C'est ce qui constitue le grand problème pour KRUGER et Lussi (I955) lorsqu'il est fait appel à du lait écrémé frais. Si, malgré cela, l'on désire valoriser de grandes quantités de lait écrémé frais pour l'élevage des veaux, on aura avantage à n'employer que des quantités restreintes de lait de remplacement.

\section{CONCLUSION}

Des expériences d'élevage sur 3 groupes de veaux mâles recevant pendant I6 semaines des quantités importantes de lait écrémé frais $(564,605$ et 6461$)$ à côté de quantités restreintes d'aliment d'allaitement (I9,3, I4, o et $8,8 \mathrm{~kg})$ offertes pendant 8,6 ou 4 semaines, nous pouvons tirer les conclusions suivantes :

Réduire la quantité d'aliment d'allaitement à $8,8 \mathrm{~kg}$ n'influence pas significativement l'augmentation en poids au cours de la période expérimentale. L'aliment d'allaitement est réparti sur une période de 4 semaines pour un régime dans lequel 6461 de lait écrémé sont administrés en 16 semaines.

Le gain de poids, supérieur en moyenne à $900 \mathrm{~g} / \mathrm{j}$ dans chacun des régimes peut être qualifié de très satisfaisant.

Des apports de faibles quantités de matières grasses semblent suffire pour garantir un bon développement. Les veaux du groupe III ne reçoivent, durant les 4 premières semaines, que $80 \mathrm{~g} / \mathrm{j}$ de matières grasses au maximum, les apports étant limités à partir de la $5^{\mathrm{e}}$ semaine à ceux fournis par les aliments concentrés et par le foin.

Lorsque, durant toute la période d'élevage, il est fait appel à des quantités de lait écrémé frais importantes, il suffira de donner, à côté du foin, un supplément d'un aliment composé à faible teneur en matières azotées et d'une composition simple (sans protéines animales) pour obtenir une bonne croissance.

Limiter la quantité de "lait de remplacement " à 4 semaines (groupe III) ou à 6 semaines (groupe II) n'entraîne pas une augmentation de la quantité d'aliments secs consommée. Le schéma alimentaire est conçu de telle sorte que les trois groupes reçoivent un même volume de produits laitiers. I'aliment d'allaitement est en effet remplacé par du lait écrémé, ce qui n'entraîne qu'une faible diminution de matière sèche et d'énergie consommée.

En prenant comme base de calcul pour les aliments employés, les prix unitaires actuellement en cours, nous avons obtenu un côtt de l'alimentation qui, par $\mathrm{kg}$ de gain, est tout aussi intéressant $( \pm 20 \mathrm{FB})$ que si les veaux étaient sevrés à un âge précoce et recevaient un régime composé de "lait de remplacement ", d'aliment 
concentré et de foin. Les besoins en main-d'œuvre qu'entraîne la distribution de quantités de lait écrémé importantes pendant une longue période, constituent toutefois un facteur défavorable pour les frais généraux d'élevage. La rentabilité d'un règime, basé sur du lait écrémé administré en abondance, est essentiellement déterminée par le prix du lait écrémé. La qualité instable du lait écrémé en période estivale, peut également donner lieu à des troubles intestinaux.

Reçu pour publication en juin 1968.

\section{REMERCIEMENTS}

Nous tenons à remercier R. VERIAEgen et G. VANOOTEGHEM pour l'aide technique qu'ils nous ont apportée dans la réalisation de ces essais.

\section{SUMMARY}

INDOOR-REARING IXXPERIMENTS WITII BUIL CALVES FED ON LIBERAI. QUANTITIES

OF FRESI SKIM MILK IN COMBINATION WITI RESTRICTED AMOUNTS OF MILK SUBSTITUTE

Three methods of calf rearing have been compared on 48 bull calves of the Red and White breeds of Eastern Flander. The calves were given with liberal quantit ies 'of skim milk for the whole rearing period : $564 \mathrm{l}, 605 \mathrm{l}, 646 \mathrm{l}$ respectively, in combination with limited amounts of milk substitute given for 8,6 or 4 weeks : $\mathrm{I} 9 \cdot 3 \mathrm{~kg}, \mathrm{i} 4 \mathrm{~kg}, 8.8 \mathrm{~kg}$ respectively. The calves were also given with a concentrate of 13.7 per cent digestible crude protein (without animal protein) up to a maximum of $3 \mathrm{~kg}$ per diay, and grass hay ad libitum.

\section{TABLE IO}

Performance (mean ialues with their standard errors) of bull calves and feed intake during 16 weeks reared on fresh skim milk, milk substitute and concentrates

\begin{tabular}{|c|c|c|c|}
\hline & Group I & Group) II & Group III \\
\hline \multirow{5}{*}{ 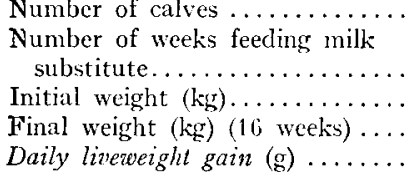 } & 16 & 16 & 16 \\
\hline & & & \\
\hline & $19.6 \pm$ & $50.1 \pm$ & $48.9 \pm$ \\
\hline & $159.6 \mp \quad 19.3$ & $151.8 \div \quad 17.7$ & $154.1+24.5$ \\
\hline & $982.1 \pm 111.8$ & $908.0 \pm 133.9$ & $939.3 \pm 181.1$ \\
\hline \multicolumn{4}{|l|}{ Feed intake (16 weeks): } \\
\hline 1. fresh skim milk (1).. & 563.64 & $605.11^{\prime}$ & $6 ; 6.19$ \\
\hline 2. milk substitute $(\mathrm{kg}) \ldots \ldots$ & 19.28 & $1 \div .03$ & 8.81 \\
\hline 3. concentrates $(\mathrm{kg}) \ldots \ldots \ldots$ & 171.86 & 166.66 & 160.86 \\
\hline 4. grasshay $(\mathrm{kg}) \ldots \ldots \ldots \ldots$ & 20.63 & 21.15 & 21.87 \\
\hline \multicolumn{4}{|l|}{ Feed cost $(F B)$ : } \\
\hline 1. per animal $(16$ weeks).... & $219 \% .91$ & 2101.27 & 2051.10 \\
\hline ?. per $\mathrm{kg}$ gain............ & 19.95 & 20.69 & 19.50 \\
\hline
\end{tabular}


The differences in liveweight gain between the three diets were not statistically significant. The average daily gain for $\mathrm{I} 2 \mathrm{2}$ days was $982 \mathrm{~g}, 908 \mathrm{~g}$ and $939 \mathrm{~g}$ respectively (table $\mathrm{ro}$ ). It appears from these results that a very small amount of fat in the ration is sufficient to obtain high growth rates. The average feed efficiency varied from $2.4 \mathrm{I}$ to 2.52 feed units per $\mathrm{kg}$ liveweight gain. Feed cost calculation based on the price levels of feedstuffs in Belgium points out a balance favorable to this rearing method (average feed cost per $\mathrm{kg}$ liveweight gain = about $3 \circ \mathrm{F} \mathrm{B}$.) However the greater demand of labour required by the administration of skim milk considerably increases the cost.

\section{RÉFÉRENCES BIBLIOGRAPHIQUES}

Boucque Ch., Buysse F., ig66. L'utilisation de différentes quantités de lait artificiel dans les régimes d'élevage de veaux femelles et l'influence de ce lait sur l'évolution ultérieure du poids de ces veaux. Revue Agric., Brux, 19, $42 \mathrm{I}-437$.

Boucque Ch., Buysse F., I967. Essai de sevrage précoce de veaux mâles destinés à la production intensive de viande. Revue Agric., Brux, 20, 415-434.

Buysse F., Martin J., i96r. Régimes d'élevage et frais d'alimentation des veaux. Revue Agric., Brux., 14, I I 57 -I I 74 .

Czako J., I964. Die Wirkung einer verminderten Milchfetternährung auf den N-Umsatz junger Kälber. Arch. Tievernähr., 14, I79-195.

EeckHout W., Buysse F., I 967 . Essais sur veaux mâles destinés à la production intensive de viande. Revue Agric., Brux., 20, 395-4I3.

Eschenbrenner G. H., I963. Untersuchungen über die Aufzucht und Mast von Jungbullen. Giessen. Schriftenreihe Tiersucht und Haustiergenetiek. 10, Verlag Paul Parey, ITamburg und Kiel.

HVidsten H., Hole G., I 962. Tilskudd av zinkbacitracin + penicillin til kalver pa moderate og store helmjölkmengder. (Zinc bacitracin + penicillin supplementation of calves on rations containing moderate and liberal quantities of whole milk). Tidsskr. norske Landbr., 69, $235^{-245}$.

Hvidsten H., Hole G., i 965 . Sammenligning av syrnet skummet mjölk med torket skummet mjölk med og uten antibiotika til kalver. (Comparison of cultured skim milk and dried skim milk fed to young calves with and without addition of antibiotics). Tidsskr. norske Landbr., 72, 31 4-323.

Kellner O., Becker M., I 966. Grundzüge der Fütterungslehre. Verlag Paul Parey, Hamburg und Berlin.

KRUGer L., Lusse W., i 955. Aufzucht und Mast von Kälbern mit verschieden hohen Gaben an Vollmilch. Futter und Fitlerung, 6, 4I 4-418.

KRUGER L., I957 a. Nährstoffbedarf und Futternormen bei Aufzucht und Mast von Jungrindern. Futter und Füllerung., 8, I 7-20.

Kruger L., I957 b. Neue Formen der Kälberaufzucht. Züchiungskunde, 29, 436-443.

Mathieu C.-H., Wegat-Litre E., i96r. Mise au point d'une méthode d'alimentation des veaux d'élevage. Ann. Zoolech., 10, I6I-I 75 .

Matineu C.-M., Wegat-Litre E., i962. Mise au point d'une méthode d'alimentation des veaux d'élevage Ann. Zoolech., 11, 197-207.

Mathiec C.-M., Barré P. E., 1964. Digestion et utilisation des aliments par le veau préruminant à l'engrais• Ann. Biol. anim., Bioch., Biophys., 4, 403-422.

OpstvedT J., ig64. Fortsatte forsök med syrna skumma mjölk til kalver. (Further research on acidified skim milk for calves). Berein. 123, jra Föringsförsökene, Norges Landbrukshögskole. I-I 9.

Richter K., Cranz K. L., Antoni J., 1957. Kälberaufzuchtversuche mit gestaffelten Vollmilchgaben. Zïchtungskunde, 29, I9I-I99.

SNedecor G. W., 1956. Statisital methods. Iowa State College Press, Ames, Iowa.

Warner R. G., FlatT W. P., Loosli J. K., 1956. Dietary factors influencing the development of the ruminant stomach. J. Agr. Food Chem., 4, 788-792. 